\title{
Brain and ventricular volume in patients with syndromic and complex craniosynostosis
}

\author{
T. de Jong • B. F. M. Rijken • M. H. Lequin • \\ M. L. C. van Veelen • I. M. J. Mathijssen
}

Received: 24 September 2011 / Accepted: 10 October 2011 /Published online: 20 October 2011

(C) The Author(s) 2011. This article is published with open access at Springerlink.com

\begin{abstract}
Purpose Brain abnormalities in patients with syndromic craniosynostosis can either be a direct result of the genetic defect or develop secondary to compression due to craniosynostosis, raised ICP or hydrocephalus. Today it is unknown whether children with syndromic craniosynostosis have normal brain volumes. The purpose of this study was to evaluate brain and ventricular volume measurements in patients with syndromic and complex craniosynostosis. This knowledge will improve our understanding of brain development and the origin of raised intracranial pressure in syndromic craniosynostosis.

Methods Brain and ventricular volumes were calculated from MRI scans of patients with craniosynostosis, 0.3 to 18.3 years of age. Brain volume was compared to age matched controls from the literature. All patient charts were reviewed to look for possible predictors of brain and ventricular volume.

Results Total brain volume in syndromic craniosynostosis equals that of normal controls, in the age range of 1 to 12 years. Brain growth occurred particularly in the first
\end{abstract}

\footnotetext{
T. de Jong $(\varangle)$ • B. F. M. Rijken • I. M. J. Mathijssen Department of Plastic and Reconstructive Surgery, and Hand Surgery, Sophia Children's Hospital, Erasmus University Medical Center,

Rotterdam, The Netherlands

e-mail: t.dejong@erasmusmc.nl

M. H. Lequin

Department of Radiology, Sophia Children's Hospital, Erasmus University Medical Center,

Rotterdam, The Netherlands
}

M. L. C. van Veelen

Department of Neurosurgery, Sophia Children's Hospital, Erasmus University Medical Center,

Rotterdam, The Netherlands
5 years of age, after which it stabilized. Within the studied population, ventricular volume was significantly larger in Apert syndrome compared to all other syndromes and in patients with a Chiari I malformation.

Conclusions Patients with syndromic craniosynostosis have a normal total brain volume compared to normal controls. Increased ventricular volume is associated with Apert syndrome and Chiari I malformations, which is most commonly found in Crouzon syndrome. We advice screening of all patients with Apert and Crouzon syndrome for the development of enlarged ventricle volume and the presence of a Chiari I malformation.

Keywords Craniosynostosis · Syndrome · Brain volume . Ventricular volume

\section{Introduction}

Children with craniosynostosis develop an abnormal head shape due to the premature closure of one or more cranial sutures. This congenital malformation occurs in one in 2100 to 2500 births. In up to $20 \%$ of these cases it is part of a syndrome, such as Apert, Crouzon, Muenke and SaethreChotzen, caused by mutations in the FGFR1, 2 and 3 and TWIST1 gene [9].

Different brain abnormalities are reported in patients with syndromic craniosynostosis including non-progressive ventriculomegaly, callosal agenesis or thinning, agenesis of the septum pellucidum, paucity of the antero-mesial temporal white matter, medial temporal lobe dysgenesis, pyramidal hypoplasia, venous malformations and Chiari I malformations $[3,4,7,8,14,15,19]$. In patients with syndromic craniosynostosis the origin of the abnormalities can either be intrinsic to the genetic defect or develop 
secondary to the craniosynostosis and associated hydrocephalus and increased intracranial pressure (ICP).

A mismatch between intracranial volume versus brain and ventricle volume is thought to be one of the causes of brain abnormalities and elevated ICP. However, in spite of the craniosynostosis the intracranial volumes are reported to be normal in patients with craniosynostosis or even enlarged in Apert and Crouzon syndrome [6, 13, 16]. Only one study reports on brain volume in syndromic craniosynostosis. They found that patients with Crouzon syndrome had a similar brain volume compared to normal controls [11]. This contradicts the assumption that a mismatch between intracranial and brain volume is the cause of raised ICP. To improve our understanding of the development of raised ICP, knowledge of brain and ventricular volume in this population is needed.

\section{Materials and methods}

Patients diagnosed with syndromic or complex craniosynostosis based on genetic testing and treated at the Dutch craniofacial center were invited to undergo MRI. Craniosynostosis was defined as complex if two or more sutures were closed and no mutation was found. The MRI were performed on a 1.5-T MR scanner (GE Healthcare, MR signa excite HD) between January 2004 and January 2011.

Brain and ventricular volumes were calculated from the transversal 3D T1 weighted MR images with the use of Brainlab $^{\circledR}$. This is a post-processing programme developed for neuronavigation. The software automatically outlines the brain and ventricle contour in each slice. If the automatic contour was questionable, it was manually edited. After outlining the brain or ventricle volume slice by slice, the processing programme automatically computes the total volume. The within-rater and between-rater reliabilities were 0.99 and 0.97 respectively.

Brain volume was compared to that in normal controls at the age of $1,4,8$ and 12 years, reported in literature $[10,12,17]$. Total ventricle volume could not be compared to that of normal controls because of the lack of normative data in the literature. A multivariate analysis was performed to look for potential predictors of brain and ventricular volume; age, gender, syndrome, Chiari I malformation and vault expansion. If patients had more than one MRI, only the first was used in the analysis, and patients with a ventriculoperitoneal shunt were excluded from the analysis. Syndromes were put in the model as dummy variables. The intraclass correlation coefficient was calculated to compare the within-rater and betweenrater reliabilities of the volume measurements. All analyses were done with SPSS 16.0 for Windows. This study was approved by the medical ethical committee of the Erasmus University (MEC2005-273).

\section{Results}

Between February 2004 and January 2011, 103 patients were invited to receive an MRI of whom 19 refused to participate. The 84 patient who received an MRI had a mean age of 8.1 years (range $0.3-18.3$ years). Of the 84 patients, 13 had Apert syndrome, 31 Crouzon syndrome, 15 Muenke syndrome, 10 Saethre-Chotzen syndrome and 15 complex craniosynostosis. The total group consisted of 44 females and 40 males. A vault expansion was performed in 66 patients prior to the MRI, at a mean age of 1.1 years. A Chiari I malformation was found in $12(14 \%)$ patients, one (8\%) patient with Apert syndrome, 10 (32\%) with Crouzon syndrome and one (7\%) with Muenke syndrome. Three patients had a ventriculoperitoneal shunt and were excluded from the ventricular volume analysis. All three had Crouzon syndrome.

The mean brain volumes at $1,4,8$ and 12 years of patients with craniosynostosis and normal controls are shown in Table 1. There was no significant difference between patients and normal controls. Age had a significant influence on brain volume $(p<0.001)$ but not on ventricular volume. The brain volume increased significantly in the first 5 years $(p=0.004)$ after which it stabilized. Patients with Apert syndrome $(p=0.004)$ had a significantly larger ventricular volume compared to all other patients. Patients with a Chiari I malformation $(p<0.001)$ had a significantly larger ventricular volume compared to patients without a Chiari I malformation. Unexpectedly, Crouzon syndrome as such was not significantly associated with ventricular volume, although most patients (10 out of 12) with a Chiari I were diagnosed with Crouzon syndrome. Patients with Crouzon syndrome and a Chiari I malformation were significantly older compared to Crouzon patients without a Chiari I malformation, the mean age being 10.1 versus 8.0 years $(p=0.018)$. Furthermore, they had a larger ventricle volume $(p=0.019)$ and were less likely to have had a vault expansion $(p=0.049)$. The syndrome-specific relation between age and total ventricular and brain volume is shown in Figs. 1 and 2.

\section{Discussion}

In this study we compared the total brain volume of patients with complex or syndromic craniosynostosis to that of normal controls from the literature. Furthermore, we looked for predictors of brain and ventricular volume. We found that the total brain volume in patients with complex or 
Table 1 Mean brain volume of patients with syndromic craniosynostosis and of normal controls

\begin{tabular}{lccc}
\hline & Craniosynostosis & Normal controls [10, 12, 17] & $p$-Value \\
\hline 1 Year & 4 & 29 & \\
$n$ & $0.90(0.43)$ & $1.06(0.03)$ & 0.048 \\
Age & $924.25(254.62)$ & $855.54(12.43)$ & 0.118 \\
Brain volume & & & \\
4 Years & 8 & 26 & 0.960 \\
$n$ & $3.95(0.60)$ & $3.96(0.52)$ & 0.166 \\
Age & $1280.88(162.05)$ & $1210.62(109.20)$ & \\
Brain volume & 16 & 20 & 0.461 \\
8 Years & $8.41(0.83)$ & $8.60(0.70)$ & 0.883 \\
$n$ & $1403.44(156.87)$ & $1391.42(23.54)$ & \\
Age & & 20 & 0.396 \\
Brain volume & 16 & $12.10(0.60)$ & 0.455 \\
12 Years & $11.92(0.60)$ & $1439.17(23.54)$ & \\
$n$ & $1464.50(148.01)$ & &
\end{tabular}

syndromic craniosynostosis is similar to that in normal controls and that ventricular volume was significantly related to Apert syndrome and the presence of a Chiari I malformation.

The majority of patients with syndromic and complex craniosynostosis have a normal or even enlarged intracranial volume, before as well as after vault expansion $[6,11$, $13,16]$. The finding that brain volume is normal suggests that the compensatory skull growth is sufficient, to allow normal brain growth. The excess of cerebrospinal fluid we observed may be the driving force behind this compensatory growth of the skull. Therefore, in these patients, raised ICP is more likely to result from raised CSF pressure than from a mismatch between intracranial and brain volume. In most patients this raised CSF pressure will have a communicating character with papilledema as the only sign [1].

Chiari I malformation is primarily seen in patients with Crouzon syndrome. In our population $32 \%$ of the patients with Crouzon syndrome had a Chiari I malformation,

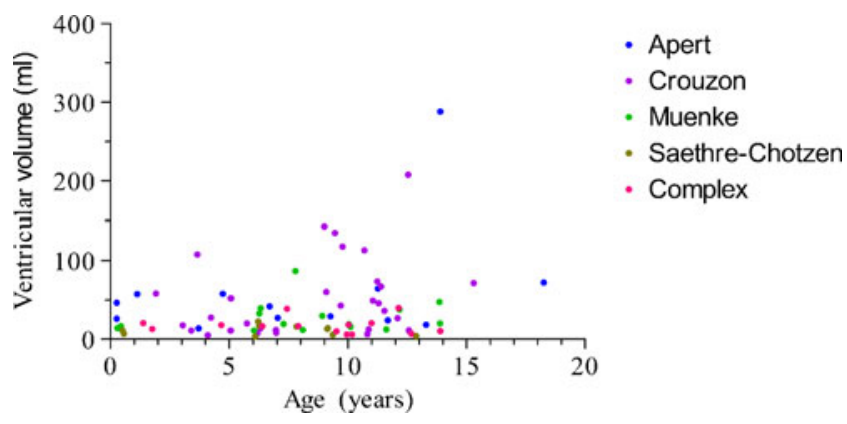

Fig. 1 Syndrome-specific relation between age and ventricular volume compared to $73 \%$ perviously reported by Cinnali et al. [2]. This difference can perhaps be explained by the fact that they performed an MRI in case of clinical signs, while we performed MRI as part of a prospective study and in most cases without a clinical indication.

The diagnosis of Crouzon syndrome itself was not associated with an enlarged ventricular volume when it was corrected for Chiari I malformation. This means that Chiari I malformations have a stronger relation with ventricular volume than Crouzon syndrome by itself. With the lack of consecutive data, we are not able to tell whether Chiari I malformation precedes or follows the enlarged ventricular volume. Enlarged ventricular volume could be the consequence of reduced CSF outflow due to Chiari I but could also be the cause of downward pressure on the cerebellum due to raised ICP. Chiari I malformations and raised ICP are both prevalent in Crouzon syndrome [18].

In Apert syndrome larger ventricles are not related to Chiari I malformation, as only 2 to $8 \%$ of the patients with Apert syndrome have a Chiari I malformation [2]. Despite

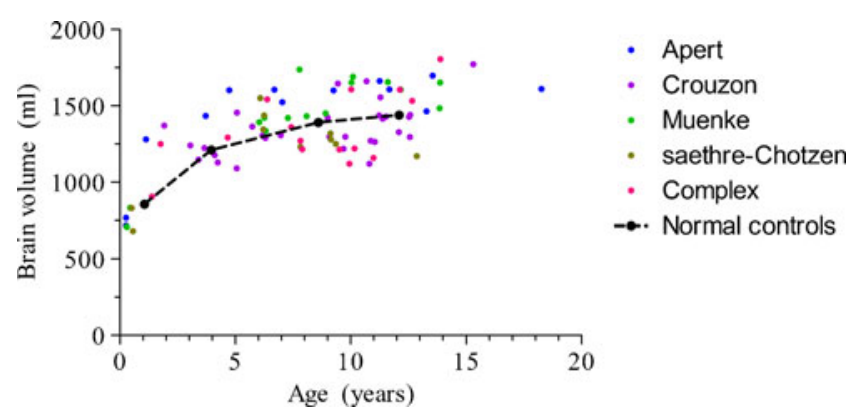

Fig. 2 Syndrome-specific relation between age and brain volume 
the larger ventricular volume, patients with Apert syndrome have a relatively low prevalence of increased ICP [5]. This could be due to their significantly larger intracranial volume before and after vault expansion $[6,16]$. In Apert syndrome extra compensatory growth of the skull is facilitated by the enlarged anterior fontanelle that stays open for a relatively long period, preventing the development of increased ICP.

\section{Conclusion}

For the first time we show that patients with syndromic and complex craniosynostosis have a normal total brain volume. Therefore, it is unlikely that a mismatch between intracranial and brain volume is the main cause of raised ICP. Furthermore, we found enlarged ventricular volume to occur particularly in patients with Apert syndrome and patients with a Chiari I malformation. Patients with Crouzon syndrome are especially at risk for Chiari I, but those without a Chiari I have normal ventricular volumes. We advice screening of all patients with Apert and Crouzon syndrome for the development of enlarged ventricle volume and the presence a Chiari I malformation.

Conflict of interest The authors declare that they have no conflict of interest.

Open Access This article is distributed under the terms of the Creative Commons Attribution Noncommercial License which permits any noncommercial use, distribution, and reproduction in any medium, provided the original author(s) and source are credited.

\section{References}

1. Bannink N, Joosten KF, van Veelen ML, Bartels MC, Tasker RC, van Adrichem LN, van der Meulen JJ, Vaandrager JM, de Jong TH, Mathijssen IM (2008) Papilledema in patients with Apert, Crouzon, and Pfeiffer syndrome: prevalence, efficacy of treatment, and risk factors. The Journal of craniofacial surgery 19(1):121-127

2. Cinalli G, Renier D, Sebag G, Sainte-Rose C, Arnaud E, Pierre-Kahn A (1995) Chronic tonsillar herniation in Crouzon's and Apert's syndromes: the role of premature synostosis of the lambdoid suture. $\mathrm{J}$ Neurosurg 83(4):575-582

3. Cinalli G, Spennato P, Sainte-Rose C, Arnaud E, Aliberti F, Brunelle F, Cianciulli E, Renier D (2005) Chiari malformation in craniosynostosis. Childs Nerv Syst 21(10):889-901

4. Collmann H, Sorensen N, Krauss J (2005) Hydrocephalus in craniosynostosis: a review. Childs Nerv Syst 21(10):902-912
5. de Jong T, Bannink N, Bredero-Boelhouwer HH, van Veelen ML, Bartels MC, Hoeve LJ, Hoogeboom AJ, Wolvius EB, Lequin MH, van der Meulen JJ, van Adrichem LN, Vaandrager JM, Ongkosuwito EM, Joosten KF, Mathijssen IM (2010) Long-term functional outcome in 167 patients with syndromic craniosynostosis; defining a syndrome-specific risk profile. J Plast Reconstr Aesthet Surg 63 (10):1635-1641

6. Gosain AK, McCarthy JG, Glatt P, Staffenberg D, Hoffmann RG (1995) A study of intracranial volume in Apert syndrome. Plast Reconstr Surg 95(2):284-295

7. Grosso S, Farnetani MA, Berardi R, Bartalini G, Carpentieri M, Galluzzi P, Mostardini R, Morgese G, Balestri P (2003) Medial temporal lobe dysgenesis in Muenke syndrome and hypochondroplasia. American journal of medical genetics 120A(1):88-91

8. Jeevan DS, Anlsow P, Jayamohan J (2008) Abnormal venous drainage in syndromic craniosynostosis and the role of CT venography. Childs Nerv Syst 24(12):1413-1420

9. Johnson D, Wilkie AO (2011) Craniosynostosis. Eur J Hum Genet 19(4):369-376

10. Knickmeyer RC, Gouttard S, Kang C, Evans D, Wilber K, Smith JK, Hamer RM, Lin W, Gerig G, Gilmore JH (2008) A structural MRI study of human brain development from birth to 2 years. J Neurosci 28(47):12176-12182

11. Mardini S, See LC, Lo LJ, Salgado CJ, Chen YR (2005) Intracranial space, brain, and cerebrospinal fluid volume measurements obtained with the aid of three-dimensional computerized tomography in patients with and without Crouzon syndrome. J Neurosurg 103(3 Suppl):238-246

12. Ment LR, Kesler S, Vohr B, Katz KH, Baumgartner H, Schneider KC, Delancy S, Silbereis J, Duncan CC, Constable RT, Makuch RW, Reiss AL (2009) Longitudinal brain volume changes in preterm and term control subjects during late childhood and adolescence. Pediatrics 123(2):503-511

13. Posnick JC, Armstrong D, Bite U (1995) Crouzon and Apert syndromes: intracranial volume measurements before and after cranio-orbital reshaping in childhood. Plast Reconstr Surg 96 (3):539-548

14. Quintero-Rivera F, Robson CD, Reiss RE, Levine D, Benson CB, Mulliken JB, Kimonis VE (2006) Intracranial anomalies detected by imaging studies in 30 patients with Apert syndrome. American journal of medical genetics 140(12):1337-1338

15. Raybaud C, Di Rocco C (2007) Brain malformation in syndromic craniosynostoses, a primary disorder of white matter: a review. Childs Nerv Syst 23(12):1379-1388

16. Sgouros S, Hockley AD, Goldin JH, Wake MJ, Natarajan K (1999) Intracranial volume change in craniosynostosis. J Neurosurg 91(4):617-625

17. Sparks BF, Friedman SD, Shaw DW, Aylward EH, Echelard D, Artru AA, Maravilla KR, Giedd JN, Munson J, Dawson G, Dager SR (2002) Brain structural abnormalities in young children with autism spectrum disorder. Neurology 59(2):184-192

18. Thompson DN, Harkness W, Jones BM, Hayward RD (1997) Aetiology of herniation of the hindbrain in craniosynostosis. An investigation incorporating intracranial pressure monitoring and magnetic resonance imaging. Pediatr Neurosurg 26(6):288-295

19. Yacubian-Fernandes A, Palhares A, Giglio A, Gabarra RC, Zanini S, Portela L, Plese JP (2004) Apert syndrome: analysis of associated brain malformations and conformational changes determined by surgical treatment. J Neuroradiol 31(2):116-122 